led to a significant increase of NF- $\kappa B$ luciferase activity in WT and all NOD2 variant constructs, except SNP13 and SNP5+13 ( $\mathrm{p}<0.0001)$. Haplotype analysis of 11 NOD2 SNPs, identified through direct sequencing in 24 children with CD, showed that LD between SNP5 and the other $\mathrm{CD}$-associated variants is low $\left(\mathrm{r}^{2}<0.1\right)$, in spite of close physical proximity (D'1.0).

Conclusion Our combined genetic and functional analyses demonstrate that the association of SNP5 with Crohn's disease is unlikely due to LD with other SNPs. At low levels of NOD2 expression, NOD2 variant constructs differ from WT in their auto-signalling and MDP-stimulated activation of NF- $\mathrm{KB}$.

Competing interests None declared.

\section{PTU-093 INAPPROPRIATE INFLAMMATORY RESPONSES IN THE ILEUM OF ULCERATIVE COLITIS PATIENTS}

\section{doi:10.1136/gutjnl-2012-302514c.93}

${ }^{1} \mathrm{~J}$ Landy, ${ }^{2} \mathrm{H} O \mathrm{Al}$-Hassi, ${ }^{1} \mathrm{~S} \mathrm{~T}$ Peake, ${ }^{2} \mathrm{~N}$ English, ${ }^{3} \mathrm{P} \mathrm{J}$ Ciclitira, ${ }^{4} \mathrm{R} \mathrm{J}$ Nicholls, ${ }^{5} \mathrm{~S}$ K Clark, ${ }^{2}$ S C Knight, ${ }^{1}$ A L Hart. ${ }^{1}$ IBD Unit, St Mark's Hospital, London, UK; ${ }^{2}$ Antigen Presentation Research Group, Imperial College, London, UK; ${ }^{3}$ Gastroenterology, St Thomas' Hospital, London, UK; ${ }^{4}$ Department of Biosurgery and Surgical Technology, Imperial College, London, UK; ${ }^{5}$ Colorectal Surgery, St Mark's Hospital, London, UK

Introduction Inflammation in ulcerative colitis (UC) is restricted to the colon. However, up to $50 \%$ of UC patients develop inflammation of the small bowel following restorative proctocolectomy (RPC). We hypothesised that in UC patients, ileal lamina propria dendritic cells would have a more "stimulatory" phenotype than in normal controls predisposing UC patients to pouch inflammation following RPC.

Methods Mucosal biopsy samples were taken from the ileum of UC patients undergoing RPC and from healthy controls undergoing colonoscopy. Lamina propria dendritic cells were isolated from biopsy tissue by collagenase digestion. DCs were identified as an HLA DR+, lineage- (CD3-, CD14-, CD16-, CD19-, CD34-, CD56-) population and expression of Toll-like receptors (TLRs), homing markers and co-stimulatory markers were measured by multicolour flow cytometry. T-tests were performed for statistical analysis.

Results There were no differences between the percentage of dendritic cells expressing TLR $2(30.5 \pm 11.5 \%$ vs $32.5 \pm 8.8 \%)$ or TLR 4 $(38.6 \pm 5.8 \%$ vs $38.8 \pm 7.2 \%)$ in the UC and healthy control ileum. A significantly greater percentage of lamina propria dendritic cells expressed the gut homing marker $\beta 7$ in the normal ileum $(33.8 \pm 9.6 \%)$ compared with the UC ileum $(6.4 \pm 3.2 \%, \mathrm{p}=0.007)$ as well as the co-stimulatory marker CD40 ( $80 \pm 2.9 \%$ vs $48.6 \pm 5.7 \%, \mathrm{p}=0.001$ ).

Conclusion Contrary to our expectations, lamina propria dendritic cells in the ileum of UC patients appear to have a less "stimulatory" phenotype than in normal controls. There may therefore be an absence of appropriate effector responses and reduced regulation by T-cells in the UC ileum. Further work is necessary to assess the T-cell responses to dendritic cell stimulation in the ileum of UC and in healthy controls.

Competing interests None declared.

\section{PTU-094 IMMUNISATION OF IBD PATIENTS ON BIOLOGIC THERAPY: AN ENGLISH DISTRICT HOSPITAL EXPERIENCE}

doi:10.1136/gutjnl-2012-302514c.94

\footnotetext{
1,2 J Kayani, ${ }^{*}{ }^{2}$ A Piotrowicz. ${ }^{1}$ East Midlands South Deanery, Leicester, UK; ${ }^{2}$ East Kent
} Hospitals University NHS Foundation Trust, Margate, UK

Introduction Patients with IBD remain at risk of preventable infections due to immunomodulatory drugs. The European Crohns and
Colitis Organisation (ECCO) Consensus document ${ }^{1}$ recommends immunising all such patients to Human Papilloma Virus (HPV-in females under 18 years), Influenza, Pneumococcus and Hepatitis B (in seronegative patients). We investigated our practice over the last 5 years.

Methods All 29 patients contactable on our Biologics' Register agreed to take part. Twenty-six remained on maintainance biologics. All had received at least one immunomodulatory drug prior to their commencement. Patients were asked if they had been immunised or offered it prior to or during their biologic therapy.

Results Responses in Abstract PTU-094 table 1. While 83\% were offered influenza immunisation-28\% declined all invitations and a similar number declined yearly offers of the immunisation. This was despite a number of pandemic flu scares. All patients reported that influenza and pneumococcal vaccination occurred at the instigation of Primary Carers. All those who received Hepatitis B immunisation did so as a result of Occupational or Travel requirement and those who were offered HPV did so as part of other national recommendations.

\section{Abstract PTU-094 Table 1}

\begin{tabular}{lllll}
\hline Vaccination & $\begin{array}{l}\text { Potential } \\
\text { recipients }\end{array}$ & Immunised (\%) & $\begin{array}{l}\text { Immunisation } \\
\text { declined (\%) }\end{array}$ & $\begin{array}{l}\text { Not offered } \\
\text { immunisation }\end{array}$ \\
\hline HPV & 2 & $1(50)$ & $1(50)$ & 0 \\
Influenza & 29 & $16(55)$ & $8(28)$ & $5(17)$ \\
Pneumococcus & 29 & $4(14)$ & 0 & $25(86)$ \\
Hepatitis B & 29 & $6(21)$ & 0 & $23(79)$ \\
\hline
\end{tabular}

Conclusion A recent survey of Australian Gastroenterologists found $30 \%-55 \%$ had never recommended such immunisations. ${ }^{2}$ Others have found that most Gastroenterologists feel that Primary carers are responsible. ${ }^{3}$ Primary carers however use National guidelines ${ }^{4}$ that have no specific recommendations for Hepatitis B and HPV in patients on immunosurpressants. Gastroenterologists therefore need to promote awareness of ECCO guidelines to ensure best coverage for patients as well as advocating standardisation in National and Professional guidelines.

Competing interests None declared.

\section{REFERENCES}

1. Rahier JF, et al. European evidence-based Consensus on the prevention, diagnosis and management of opportunistic infections in inflammatory bowel disease. Journal of Crohn's and Colitis 2009;3:47-91.

2. Gupta A, Macrae FA, Gibson PR. Vaccination and screening for infections with inflammatory bowel disease: a survey of Australian gastroenterologists. Internal Medicine Journal 2011;41:462-7.

3. Wasan SK, Skolnik PR, Farraye FA. A 24 Year Old patient with Crohn's disease starting immunosurpressive therapy: vaccination issues to consider. Clin Gastroenterol Hepatol 2010;8:1013-16

4. Chapter 7 in Immunisations against infectious disease (the Green Book)-UK Dept of Health Online Guidelines. 2006 (Nov 2011 update).

\section{PTU-095 A QUALITATIVE EXPLORATION OF INFLAMMATORY BOWEL DISEASE PATIENT PERCEPTIONS OF PRIMARY CARE IN THE UK}

doi:10.1136/gutjnl-2012-302514c.95

${ }^{1} \mathrm{~K}$ Kemp, ${ }^{*}{ }^{1} \mathrm{~J}$ Griffiths, ${ }^{2} \mathrm{~S}$ Campbell, ${ }^{1} \mathrm{~K}$ Lovell. ${ }^{1}$ School of Nursing, University of Manchester, Manchester, UK; ${ }^{2}$ Gastroenterology, Manchester Royal Infirmary, Manchester, UK

Introduction Many patients with IBD in the UK are managed primarily in secondary care with minimal General Practitioner (GP) involvement or within a restricted shared care protocol. As UK NHS hospitals face mounting financial and workforce pressures, 
reconfiguration of hospital services can provide a powerful means of improving the quality of patient care. The issue as to whether GPs should be more involved with IBD care must be addressed and the patient's attitudes and perceptions of the GP and potential role, should be a focus when restructuring healthcare services.

Methods 24 in depth qualitative interviews with 24 IBD patients were conducted as part of a larger study to develop a model of follow-up care for patients with IBD. 18 patients had Crohns Disease, 6 ulcerative colitis, age range $27-72$ years, disease duration range was $2-40$ yrs. Interviews were 40 to 60 min duration. Patients were asked directly about the role of their GP in their diagnosis and ongoing care of their IBD. Thematic analysis of interviews was undertaken using NVivo 9.0.

Results Many patients experienced years of symptoms prior to diagnosis, in one case diagnosis took 10 years. This misdiagnosis/ mistreatment led to a loss of confidence in the GP for future care. This impacted upon the question if they seek help from their GP at times of flares or IBD related problems. All patients reported no they do not seek help from their GP. This loss of confidence at diagnosis promoted views that their GP did not have the level of knowledge nor expertise to manage ongoing care. This was the same for patients with complex and quiescent disease. Patients would be happy to increase the level of input from their GP but this must be under the direction of the IBD team. Patients do not wish to be discharged completely from the IBD team and would accept reduced intervention and face to face contact with their IBD team, such as telephone and virtual clinics, in order to remain under the care of the IBD team.

Conclusion GPs are the sole carers for a minority of IBD patients. While patients are willing to accept greater involvement from their GP there is general lack of confidence in their expertise to do this effectively. This is reflected by patients expressing a wish to remain under the direct care of the IBD team within secondary care. It is clear from the data that there may be an educational developmental need for the GPs regarding IBD but there is potential for reconfiguration of IBD services in which the GP plays a greater role.

Competing interests None declared.

\section{PTU-096 THE USE OF COMMERCIAL INTERFERON- $\gamma$ RELEASE ASSAYS TO SCREEN FOR MYCOBACTERIAL INFECTION IN INFLAMMATORY BOWEL DISEASE PATIENTS INITIATING ANTI-TNF AGENTS}

doi:10.1136/gutjnl-2012-302514c.96

${ }^{1} \mathrm{~K}$ Greveson, ${ }^{1} \mathrm{C}$ Murray, ${ }^{1} \mathrm{~J}$ Goodhand, ${ }^{2} \mathrm{C}$ Cropley, ${ }^{3} \mathrm{~S}$ Murthy, ${ }^{1} \mathrm{M}$ Hamilton, ${ }^{3} \mathrm{M}$ Lipman. ${ }^{1}$ Centre for Gastroenterology, Royal Free Hospital, London, UK; ${ }^{2}$ Infectious Diseases, Royal Free Hospital, London, UK; ${ }^{3}$ Respiratory Medicine, Royal Free Hospital, London, UK

Introduction Two commercial Interferon Gamma release assays (IGRA) are approved in the UK by NICE and US FDA to detect $M$ tuberculosis (Mtb) infection. The T-Spot.TB (TSTB) and Quanitferon Gold In-tube (OFGIT) use different test platforms, with the potential for slightly different results when used in clinical practice. Since 2008, we have used a standard IGRA-based assessment for Mtb infection in inflammatory bowel disease (IBD) patients. Initially this involved TSTB but in December 2010, switched to OFGIT. Here we review the performance of these assays within our protocol.

Methods We prospectively screened 148 adult IBD patients considered for anti-TNf $\alpha$ agents with symptom review, chest radiograph and IGRA. Between October 2008 and November 2010, 91 patients were tested with TSTB, and between December 2010 and November 2011, 57 with OFGIT. IGRA results were reported as positive, negative or indeterminate. Positive and indeterminate results were referred to $\mathrm{TB}$ services.

Results All subjects had normal chest radiographs and a negative clinical assessment. Overall 82\% (121/148) subjects tested were BCG vaccinated and 13\% (19/148) had risk factors for Mtb. 74\% (109/ 148) were taking immunomodulators. $98 \%(89 / 91)$ patients in the TSTB group had an unequivocal result [1\% (1/91) positive: $97 \%$ (88/ 91) negative], and $1 \%(1 / 91)$ had an indeterminate result, compared with $86 \%$ unequivocal [0\% positive/86\% negative] and $14 \%(8 / 57)$ indeterminate in the OFGIT group, respectively $(p=0.002) .12 \%$ indeterminate results occurred in subjects taking immunosuppression. $85 \%(126 / 148)$ of the anti-TNF $\alpha$ naive group have subsequently received treatment with either infliximab or adalimumab. None have gone on to develop tuberculosis. Median follow-up from start of therapy in the TSTB group is 21 months (IOR 15-26 months); and that of the OFGIT group 7 months (IOR 4-9).

Conclusion We find little evidence for Mtb infection within our IBD population. To date none have developed active TB after starting anti-TNF $\alpha$ therapy. Given the reasonable median follow-up (21 months for TSTB and 7 months for QFGIT) compared to the reported time of onset of active TB following anti-TNF $\alpha$ agents ( $<3$ months), our data provide some reassurance that we have not missed LTBI using our assessment. There appears to be a higher frequency of indeterminate results using OFGIT. As this is found almost exclusively in those on immunosuppressive agents, we suggest that IBD services need to understand the characteristics of the IGRA used within their population and the implications of this for management.

Competing interests None declared.

\section{PTU-097 MEDICAL RESOURCE UTILISATION AND COSTS IN PATIENTS WITH ULCERATIVE COLITIS IN THE UK: A CHART REVIEW ANALYSIS}

doi:10.1136/gutjnl-2012-302514c.97

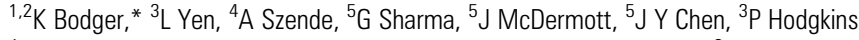
${ }^{1}$ Digestive Diseases Centre, Aintree University Hospital, Liverpool, UK; ${ }^{2}$ Department of Gastroenterology, Institute of Translational Medicine, University of Liverpool, Liverpool, UK; ${ }^{3}$ Shire Development LLC, Wayne, USA; ${ }^{4}$ Covance Market Access Services, Leeds, UK; ${ }^{5}$ Covance Market Access Services, Gaithersburg, USA
\end{abstract}

Introduction Limited evidence is available on the economic burden of ulcerative colitis (UC) in the UK, particularly in relation to the impact of relapse frequency on direct medical costs. The objective of this study was to quantify annual disease-related and all-cause (total) medical resource utilisation (MRU) and associated direct medical costs.

Methods A retrospective chart review of patients with mild-tomoderate UC diagnosed at least 1 year prior to the study was performed. From 33 general practitioner (GP) and 34 gastroenterologist sites in UK, charts of the last three UC patients meeting the study's inclusion criteria were reviewed. 2009-2010 National Health Service reference costs were assigned to the recorded medical resources. Descriptive statistics were calculated for disease-related and all-cause MRU and costs by number of relapses. Logistic regression was used to estimate MRU and costs while accounting for relapse status, patient demographics, site type, and treatment setting.

Results Study population: N=201 patients; mean age: 39.9 years; $44 \%$ female; mean disease duration: 7.4 years. UC-related costs of each MRU category increased with the number of relapses. Comparing those without relapse to patients with $>2$ relapses, mean annual UC-related costs were $£ 14$ vs $£ 2556$ for hospitalisations; $£ 239$ vs $£ 2221$ for visits; $£ 21$ vs $£ 1303$ for procedures; $£ 16$ vs $£ 188$ for diagnostics. Age, gender, and site of data reporting (GP vs gastroenterologist) were not related to MRU or costs. 